\title{
Nodeless superconductivity in the kagome metal $\mathrm{CsV}_{3} \mathrm{Sb}_{5}$
}

\author{
Weiyin Duan, ${ }^{1,2}$ Zhiyong Nie, ${ }^{1,2}$ Shuaishuai Luo, ${ }^{1,2}$ Fanghang Yu, ${ }^{3}$ Brenden R. Ortiz, ${ }^{4}$ \\ Lichang Yin, ${ }^{1,2}$ Hang Su, ${ }^{1,2}$ Feng Du, ${ }^{1,2}$ An Wang, ${ }^{1,2}$ Ye Chen, ${ }^{1,2}$ Xin Lu, ${ }^{1,2}$ Jianjun \\ Ying, ${ }^{3}$ Stephen D. Wilson, ${ }^{4}$ Xianhui Chen, ${ }^{3,5,6}$ Yu Song,, , , * and Huiqiu Yuan ${ }^{1,2,6,7, \text { 丹 }}$ \\ ${ }^{1}$ Center for Correlated Matter and Department of Physics, Zhejiang University, Hangzhou 310058, China \\ ${ }^{2}$ Zhejiang Province Key Laboratory of Quantum Technology and Device, \\ Department of Physics, Zhejiang University, Hangzhou 310058, China \\ ${ }^{3}$ Hefei National Laboratory for Physical Sciences at Microscale and Department of Physics, \\ and CAS Key Laboratory of Strongly-coupled Quantum Matter Physics, \\ University of Science and Technology of China, Hefei, Anhui 230026, China \\ ${ }^{4}$ Materials Department and California Nanosystems Institute, \\ University of California Santa Barbara, Santa Barbara, CA, 93106, United States \\ ${ }^{5}$ CAS Center for Excellence in Quantum Information and Quantum Physics, Hefei, Anhui 230026, China \\ ${ }^{6}$ Collaborative Innovation Center of Advanced Microstructures, Nanjing 210093, People's Republic of China \\ ${ }^{7}$ State Key Laboratory of Silicon Materials, Zhejiang University, Hangzhou 310058, China
}

(Dated: March 23, 2021)

\begin{abstract}
The recently discovered kagome metal series $A \mathrm{~V}_{3} \mathrm{Sb}_{5}(A=\mathrm{K}, \mathrm{Rb}, \mathrm{Cs})$ exhibits topologically nontrivial band structures, chiral charge order and superconductivity, presenting a unique platform for realizing exotic electronic states. The nature of the superconducting state and the corresponding pairing symmetry are key questions that demand experimental clarification. Here, using a technique based on the tunneling diode oscillator, the magnetic penetration depth $\Delta \lambda(T)$ of $\mathrm{CsV}_{3} \mathrm{Sb}_{5}$ was measured down to $0.07 \mathrm{~K}$. A clear exponential behavior in $\Delta \lambda(T)$ with marked deviations from a $T$ or $T^{2}$ temperature dependence is observed at low temperatures, indicating a deficiency of nodal quasiparticles. Temperature dependence of the superfluid density and electronic specific heat can be described by two-gap $s$-wave superconductivity, consistent with the presence of multiple Fermi surfaces in $\mathrm{CsV}_{3} \mathrm{Sb}_{5}$. These results evidence nodeless superconductivity in $\mathrm{CsV}_{3} \mathrm{Sb}_{5}$ under ambient pressure, and constrain the allowed pairing symmetry.
\end{abstract}

PACS numbers: 74.25.Ha, 74.70.-b, 78.70.Nx

The unique geometry of the kagome lattice leads to magnetic frustration [1 $[3]$, topologically nontrivial electronic structures [4 6 ], and both electronic [6 8] and magnon [9] flat bands. Superconductivity with exotic pairing symmetries and properties were also predicted for the kagome lattice 10 14], although physical realizations of such exotic superconductors have been limited.

The recent discovery of superconductivity in the twodimensional kagome metal series $A \mathrm{~V}_{3} \mathrm{Sb}_{5}(A=\mathrm{K}, \mathrm{Rb}$, $\mathrm{Cs}$ ) provides a much-desired platform to investigate potentially exotic superconducting states on the kagome lattice [15 18]. In addition to superconductivity, these systems also exhibit a chiral charge order with unusual characteristics 19 24, topological band crossings [15, 16], and a giant anomalous Hall effect in the absence of magnetic local moments [16, 25 27]. The nature of the superconducting state in the presence of these electronic states, remains to be clarified. Nonetheless, tantalizing evidence for an unusual superconducting state has emerged from the observation of spin-triplet supercurrent in $\mathrm{K}_{1-x} \mathrm{~V}_{3} \mathrm{Sb}_{5}$.Josephson junctions [28] and possible Majorana bound states inside the superconducting vortex cores of $\mathrm{CsV}_{3} \mathrm{Sb}_{5}[29]$.

A hallmark of unconventional superconductivity is a sign-changing superconducting order parameter [30, 31]. While direct phase-sensitive evidence for such a sign- change is difficult to obtain [32, 33], for a number of pairing symmetries the sign-change mandates nodes in the superconducting order parameter (such as $d_{x^{2}-y^{2-}}$ pairing in the cuprates), and can be probed through various experimental techniques. Thermal conductivity measurements on $\mathrm{CsV}_{3} \mathrm{Sb}_{5}$ down to $0.15 \mathrm{~K}$ indicated possible nodal quasiparticles 34, and superconducting domes were found in the temperature-pressure phase diagrams of $A \mathrm{~V}_{3} \mathrm{Sb}_{5}$ 34 36, reminiscent of behaviors in established unconventional superconductors. On the other hand, the tunneling spectrum of $\mathrm{CsV}_{3} \mathrm{Sb}_{5}$ measured by scanning tunneling microscopy could be described by an anisotropic $s$-wave gap without nodes 29$]$. To further clarify the pairing symmetry in the $A \mathrm{~V}_{3} \mathrm{Sb}_{5}$ series, experiments sensitive to low-energy excitations are badly needed.

In this work, the magnetic penetration depth and specific heat of the kagome metal $\mathrm{CsV}_{3} \mathrm{Sb}_{5}$ were measured to probe its superconducting gap structure. The change of the magnetic penetration depth reveals a clear exponential behavior down to $0.07 \mathrm{~K}$, and deviates significantly from a $T$ or $T^{2}$ temperature dependence. Such a behavior suggests an absence of nodal quasiparticles, and instead points to fully-gapped superconductivity in $\mathrm{CsV}_{3} \mathrm{Sb}_{5}$ under ambient pressure. By analyzing the derived superfluid density and electronic specific heat, a two-gap $s$ - 

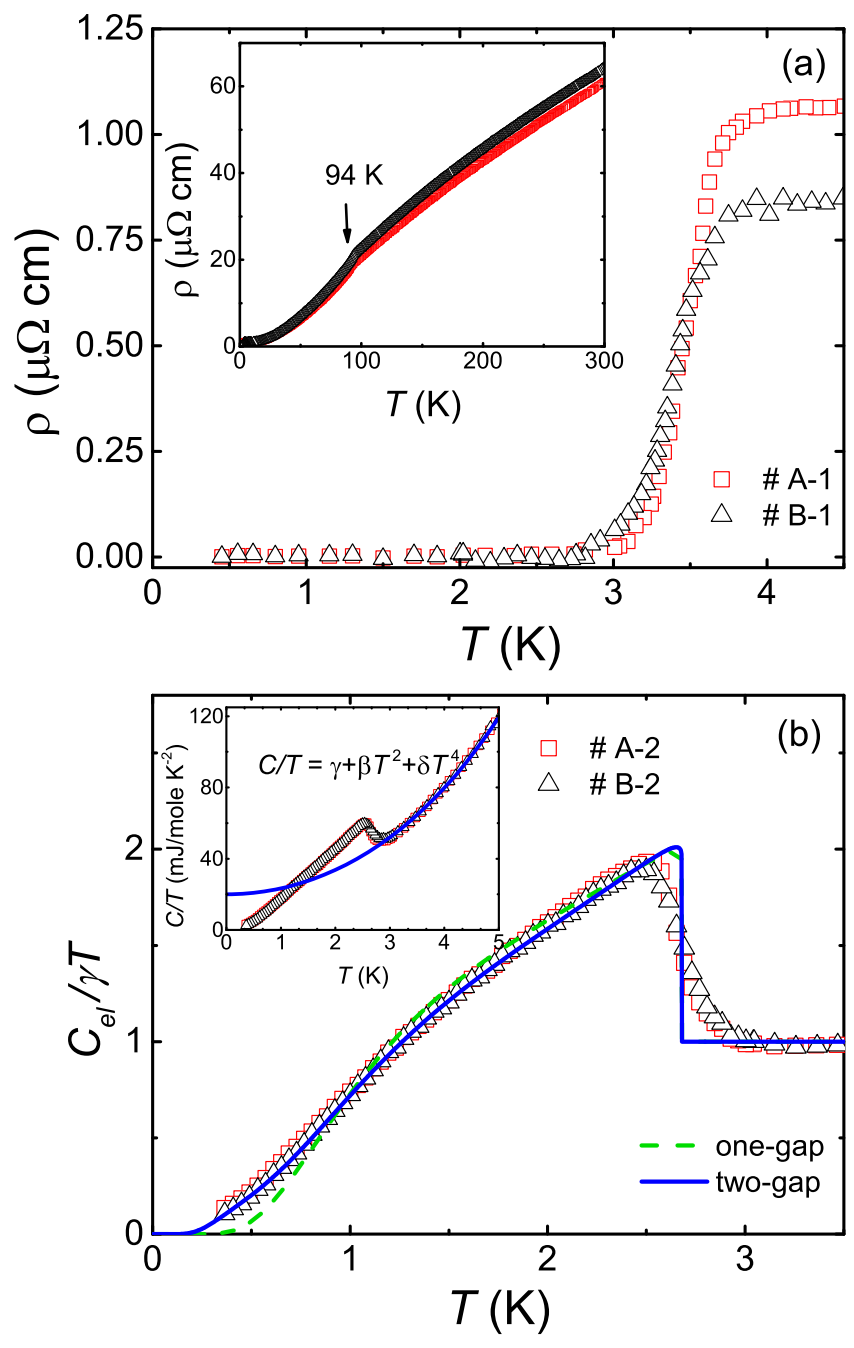

Figure 1: (Color online) Temperature dependence of the low temperature (a) resistivity $\rho(T)$ and (b) electronic specific heat $C_{\mathrm{el}}(T) / \gamma T$ for $\mathrm{CsV}_{3} \mathrm{Sb}_{5}$. The inset in (a) shows $\rho(T)$ from $0.45 \mathrm{~K}$ up to $300 \mathrm{~K}$. The inset in (b) shows the specific heat $C(T) / T$, with the blue solid line being a fit to the normal state specific heat, including contributions from electrons and phonons. The solid and dashed lines in (b) are fits to a singlegap and two-gap $s$-wave superconducting model, respectively.

wave superconducting order parameter is found to capture the experimental data. These results provide evidence for nodeless superconductivity in $\mathrm{CsV}_{3} \mathrm{Sb}_{5}$ under ambient pressure, and rule out pairing with symmetryenforced nodes.

Single crystals of $\mathrm{CsV}_{3} \mathrm{Sb}_{5}$ were synthesized using the self-flux method, at the University of Science and Technology of China (sample A) and the University of California, Santa Barbara (sample B), with physical properties separately characterized and described in previous works 16, 27]. Eight samples were examined with various approaches, and are labeled samples \#A-1 to \#A-5, and samples \#B-1 to \#B-3. The $a b$-plane electrical resistivity $\rho(T)$ was measured in a ${ }^{3} \mathrm{He}$ cryostat, using a standard four-probe method. Specific heat was measured using a
Quantum Design Physical Property Measurement System (PPMS) with a ${ }^{3} \mathrm{He}$ insert, using a standard pulse relaxation method. The change in the London penetration depth $\Delta \lambda(T)=\lambda(T)-\lambda(0)$ was measured using a tunnel diode oscillator (TDO) 37, 38], with an operating frequency of about $7 \mathrm{MHz}$. The TDO method can precisely measure the temperature dependence of the magnetic penetration depth change, offering a powerful probe of low-energy excitations in the superconducting state. TDO measurements down to $0.35 \mathrm{~K}$ and $0.07 \mathrm{~K}$, with noise levels as low as $0.1 \mathrm{~Hz}$ and $0.5 \mathrm{~Hz}$, were carried out in ${ }^{3} \mathrm{He}$ and dilution refrigerators, respectively. The ac field generated by the coil $(20 \mathrm{mOe})$ is far below the lower critical field $H_{\mathrm{c} 1}$ of $\mathrm{CsV}_{3} \mathrm{Sb}_{5}$, ensuring that the sample remains in the full Meissner state throughout the measurements. $\Delta \lambda(T)$ can be obtained from the frequency shift $\Delta f(T)$ of the TDO through $\Delta \lambda(T)=G \Delta f(T)$, where $G$ is a sample-dependent scale factor [39].

The inset of Fig. 1(a) shows the electrical resistivity $\rho(T)$ of $\mathrm{CsV}_{3} \mathrm{Sb}_{5}$ from $300 \mathrm{~K}$ down to $0.45 \mathrm{~K}$. A clear kink can be observed around $94 \mathrm{~K}$, due to the onset of charge order [15, 16]. The temperature evolution of $\rho(T)$ for samples \#A-1 and \#B-1 are highly similar, with residual resistivity ratios $(\mathrm{RRR})$ of $\approx 57$ and $\approx 74$, respectively. Fig. 1(a) zooms into $\rho(T)$ below $4.5 \mathrm{~K}$, where a clear superconducting transition can be observed, which onsets around $3.5 \mathrm{~K}$ and zero resistance appears below $\approx 2.7 \mathrm{~K}$ in both samples. The residual resistivities just above the onset of superconductivity are also similar, close to $1 \mu \Omega \mathrm{cm}$ in both samples. Measurements of the low temperature specific heat $C(T) / T$ are shown in the inset of Fig. 1(b) for samples \#A-2 and \#B-2, demonstrating the appearance of bulk superconductivity below $T_{\mathrm{c}} \approx 2.7 \mathrm{~K}$. In the normal state, the specific heat can be modeled using $C(T) / T=\gamma+\beta T^{2}+\delta T^{4}$, with $\gamma=20.03 \mathrm{~mJ} \mathrm{~mole}^{-1} \mathrm{~K}^{-2}, \beta=3.306 \mathrm{~mJ} \mathrm{~mole}^{-1} \mathrm{~K}^{-4}$ and $\delta=26.78 \mu \mathrm{J} \mathrm{mole}{ }^{-1} \mathrm{~K}^{-6}$ (solid line in the inset of Fig. T(b)). Here $\gamma$ is the Sommerfeld coefficient, and the other two parameters characterize the contribution from phonons. After subtracting the phonon contribution, the electronic specific heat $C_{\mathrm{el}}(T) / \gamma T$ can be obtained, shown in Fig. T(b). Analysis of $C_{\mathrm{el}}(T) / \gamma T$ will be further discussed below. Combining the coherence length $\xi\left(\approx 26 \mathrm{~nm}\right.$ from $\left.H_{\mathrm{c} 2}=0.47 \mathrm{~T}[16,27]\right)$, residual resistivity $\rho_{0}$, and the Sommerfeld coefficient $\gamma$, mean free paths of $680 \mathrm{~nm}$ and $830 \mathrm{~nm}$ are estimated [40, 41] for samples \#A-1 and \#B-1, respectively. These values are much larger than the coherence length, indicating that the samples are in the clean limit. Our resistivity and specific heat results are consistent with previous reports [16, 27], and indicate samples A and B are similar and that both are of high quality.

Fig. 2 shows the temperature dependence of the magnetic penetration depth for $\mathrm{CsV}_{3} \mathrm{Sb}_{5}$. Three samples were studied with applied field along the $c$-axis, with $G=7.8 \AA / \mathrm{Hz}, 9.1 \AA / \mathrm{Hz}$ and $9.8 \AA / \mathrm{Hz}$, for samples 
\#A-3, \#A-4 and \#B-3, respectively. Sample \#A-5 was studied with field perpendicular to the $c$-axis, with its $\Delta \lambda(T)$ scaled to that of sample \#A-3, due to difficulties in accurately determining the $G$ factor for a thin sample. The inset in Fig. 2(a) shows $\Delta \lambda(T)$ for samples \#A-3 and \#A-5 from $4.5 \mathrm{~K}$ down to $0.07 \mathrm{~K}$, exhibiting clear reductions upon cooling due to superconductivity, consistent with resistivity and specific heat measurements in Fig. 1. $\Delta \lambda(T)$ with applied field along and perpendicular to the $c$-axis are compared in Fig. 2(a) for samples \#A-3 and \#A-5, revealing almost identical behaviors, suggesting that the superconducting state is rather isotropic. Fig. 2(b) compares the low temperature penetration depth $\Delta \lambda(T)$ for three $\mathrm{CsV}_{3} \mathrm{Sb}_{5}$ samples with field applied along the $c$-axis. The data for different samples almost overlap, confirming the superconducting properties of samples A and B are similar, and demonstrate that our results are reproducible and reflect the intrinsic behavior of $\mathrm{CsV}_{3} \mathrm{Sb}_{5}$.

For a nodal superconductor in the clean limit, it is expected that the magnetic penetration depth exhibits a power-law behavior in the low temperature limit, i.e., $\Delta \lambda \sim T^{n}$, with $n=1$ and 2 respectively corresponding to line nodes and point nodes being present in the gap structure. From Fig. 2(b), it can be seen that the experimentally measured $\Delta \lambda(T)$ obviously deviates from a $T$ or $T^{2}$ behavior, but is reasonably described by $\Delta \lambda(T) \sim T^{2.9}$ with a slight deviation at low temperatures. To further analyze the power law dependence of $\Delta \lambda(T)$, the experimental data is fit by $\Delta \lambda(T) \sim T^{n}$ from $0.07 \mathrm{~K}$ to various temperatures, with the best fit $n$ shown in the inset of Fig. 2(b). It can be seen that the exponent $n \approx 3$ appears for $T>0.2 T_{\mathrm{c}}$, and becomes significantly enhanced at lower temperatures. Such a power law behavior $(n \gtrsim 3)$, in particular the progressive increase of $n$ with decreasing temperature, is consistent with an exponential behavior, and suggests an absence of gap nodes in the superconducting state of $\mathrm{CsV}_{3} \mathrm{Sb}_{5}$.

To further analyze the magnetic penetration depth, $\Delta \lambda(T)$ is fit to an $s$-wave gap at low temperatures, with

$$
\Delta \lambda(T) \sim T^{-\frac{1}{2}} \exp \left(-\frac{\Delta(0)}{k_{\mathrm{B}} T}\right),
$$

where $\Delta(0)$ is the gap value at zero temperature. It can be seen that such an $s$-wave model fits the experimental data well at low temperatures, providing strong evidence for nodeless superconductivity in $\mathrm{CsV}_{3} \mathrm{Sb}_{5}$. The derived small superconducting gap of $\Delta(0)=0.59 k_{\mathrm{B}} T_{\mathrm{c}}$ indicates that $\Delta \lambda(T)$ would only saturate at very low temperatures, as seen in Fig. 2(b), and a multi-gap model is needed in order to describe $\Delta \lambda(T)$.

To further extract information about the superconducting state, the normalized superfluid density is obtained through $\rho_{s}(T)=[\lambda(0) / \lambda(T)]^{2}$. The value of the zero-temperature penetration depth $\lambda(0)=387 \mathrm{~nm}$ was estimated using $\lambda(0)=\sqrt{\phi_{0} H_{\mathrm{c} 2}(0)} / \sqrt{24 \gamma} \Delta(0)$ [42],
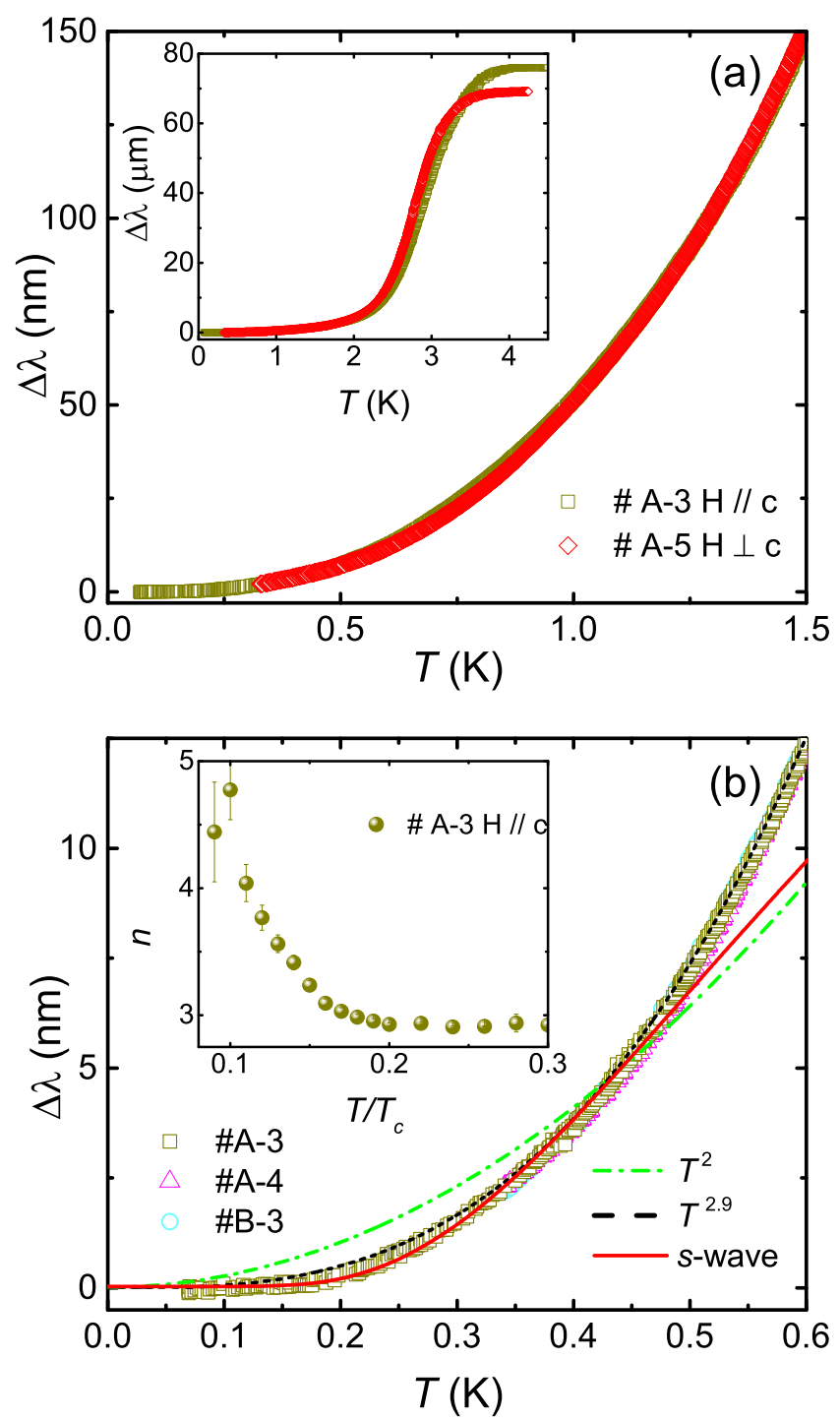

Figure 2: (Color online) (a) Comparison of $\Delta \lambda(T)$ for applied fields along and perpendicular to the $c$-axis. The inset shows $\Delta \lambda(T)$ from $4.5 \mathrm{~K}$ down to $0.07 \mathrm{~K}$. (b) The change of the penetration depth $\Delta \lambda(T)$, measured for three $\mathrm{CsV}_{3} \mathrm{Sb}_{5}$ samples with field along the $c$-axis. The dash-dotted and dashed lines respectively represent fits using a $T^{2}$ and a $T^{n}$ temperature dependence, from $0.07 \mathrm{~K}$ up to $0.6 \mathrm{~K}$. The solid lines represents a fit to Eq. 1 The inset shows the exponent $n$, obtained by fitting $\Delta \lambda(T)$ to a $T^{n}$ behavior from $0.07 \mathrm{~K}$ up to different temperatures of $T / T_{\mathrm{c}}$.

where $\phi_{0}$ is the magnetic-flux quantum, the Sommerfeld coefficient $\gamma$ is obtained from specific heat measurements (inset of Fig. 1](b)), $H_{\mathrm{c} 2}(0)=0.47 \mathrm{~T}$ [16, 27] and the weak-coupling limit of BCS theory with $\Delta(0)=$ $1.76 k_{\mathrm{B}} T_{\mathrm{c}}$ is assumed. $\rho_{\mathrm{s}}$ obtained this way is shown as a function of the reduced temperature $T / T_{\mathrm{c}}$ for sample \#A-3 in Fig. 3(a), and is fit to several different models of the superconducting gap function $\Delta_{k}$, which is related to $\rho_{\mathrm{s}}$ through 


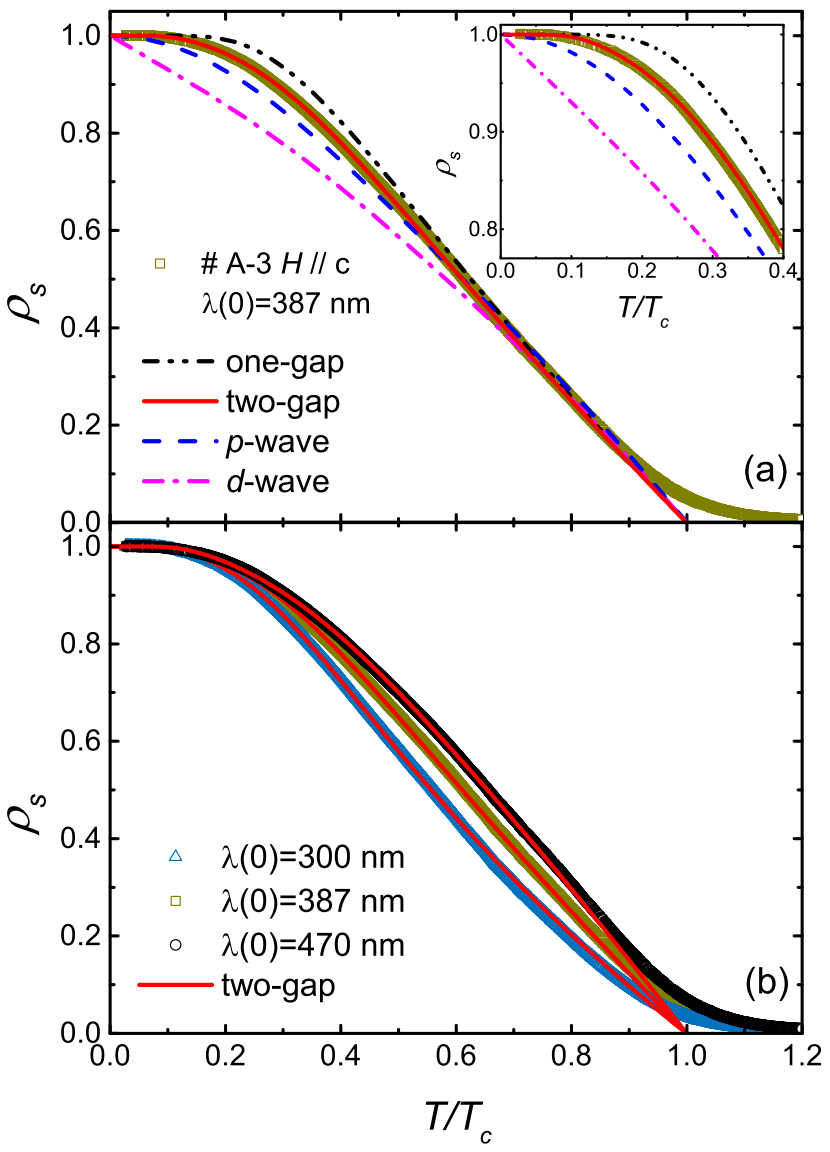

Figure 3: (Color online) Normalized superfluid density $\rho_{\mathrm{s}}$ for sample \#A-3 with $\lambda(0)=387 \mathrm{~nm}$, as a function of the reduced temperature $T / T_{\mathrm{c}}$. The dash-dot-dotted, solid, dashed, and dash-dotted lines respectively represent fits to models with a single $s$-wave gap, two $s$-wave gaps, a $p$-wave gap, and a $d$ wave gap. The inset zooms into the low temperature region of the figure. (b) Comparison of $\rho_{\mathrm{s}}$ for different values of $\lambda(0)$. The solid lines are fits to a two-gap $s$-wave model.

$$
\rho_{\mathrm{S}}(T)=1+2\left\langle\int_{\Delta_{k}}^{\infty} \frac{E \mathrm{~d} E}{\sqrt{E^{2}-\Delta_{k}^{2}}} \frac{\partial f}{\partial E}\right\rangle_{\mathrm{FS}},
$$

where $f(E, T)=\left[1+\exp \left(\frac{E}{k_{\mathrm{B}} T}\right)\right]^{-1}$ is the Fermi-Dirac distribution, and $\langle\ldots\rangle_{\mathrm{FS}}$ represents averaging over the Fermi surface. Temperature dependence of the superconducting order parameter is approximated using

$$
\Delta(T)=\Delta(0) \tanh \left\{1.82\left[1.018\left(T_{\mathrm{c}} / T-1\right)\right]^{0.51}\right\}
$$

As can be seen, the data are clearly incompatible with a single-gap $s$-wave model, a $p$-wave model with point nodes, and a $d$-wave model with line nodes. These models exhibit significant deviations from the data, especially at low temperatures (inset of Fig. 3(a)). On the other hand, a two-gap $s$-wave model describes the data well over the entire temperature range (solid line in Fig. 3. a)). The fit gap values are $\Delta_{1}(0)=1.42 k_{\mathrm{B}} T_{\mathrm{c}}$ and $\Delta_{2}(0)=0.58 k_{\mathrm{B}} T_{\mathrm{c}}$, with weights of the two gaps being $87 \%$ and $13 \%$, respectively. The deduced small gap of $\Delta_{2}=0.58 k_{\mathrm{B}} T_{\mathrm{c}}$ is consistent with $\Delta=0.59 k_{\mathrm{B}} T_{\mathrm{c}}$ derived from fitting $\Delta \lambda(T)$ at low-temperature (Fig. 2(b)). Considering possible uncertainties in the estimate of $\lambda(0)$ and to test the robustness of our conclusions, $\lambda(0)$ is varied by $\approx \pm 20 \%$ from $387 \mathrm{~nm}$ and the analyses are repeated, with results shown in Fig. 3(b). It is found that the two-gap $s$-wave model describes $\rho_{\mathrm{s}}$ well in all cases (solid lines), while other models in Fig. 3(a) exhibit clear deviations. For $\lambda(0)=300 \mathrm{~nm}$, $\Delta_{1}(0)=1.23 k_{\mathrm{B}} T_{\mathrm{c}}$ and $\Delta_{2}(0)=0.50 k_{\mathrm{B}} T_{\mathrm{c}}$ are obtained, while $\Delta_{1}(0)=1.63 k_{\mathrm{B}} T_{\mathrm{c}}$ and $\Delta_{2}(0)=0.66 k_{\mathrm{B}} T_{\mathrm{c}}$ are obtained for $\lambda(0)=470 \mathrm{~nm}$. Our analyses of the superfluid density therefore support the idea that $\mathrm{CsV}_{3} \mathrm{Sb}_{5}$ exhibits multiband nodeless superconductivity. It is noted that the larger gap $\Delta_{1}(0)$ is close to but still smaller than the BCS value of $1.76 k_{\mathrm{B}} T_{\mathrm{c}}$, the origin of which is not clear. It is possible that the contribution of an additional band or the effect of an anisotropic gap may lead to such a weak deviation.

To confirm the above conclusion, the low temperature electronic specific heat $C_{\mathrm{el}}(T) / \gamma T$ of $\mathrm{CsV}_{3} \mathrm{Sb}_{5}$ is analyzed, shown in Fig. 1(b). Similar to the superfluid density, it is found that a single $s$-wave gap does not capture the behavior of $C_{\mathrm{el}}(T) / \gamma T$ (solid line in Fig. 1(b)), while a two-gap $s$-wave model gives an excellent description of the experimental data over the full temperature range (dashed line in Fig. 1(b)). The fit gap values are $\Delta_{1}(0)=1.62 k_{\mathrm{B}} T_{\mathrm{c}}$ and $\Delta_{2}(0)=0.63 k_{\mathrm{B}} T_{\mathrm{c}}$, with weights of $79 \%$ and $21 \%$, respectively. These values are in reasonable agreement with analysis of the superfluid density, and supports the notion that $\mathrm{CsV}_{3} \mathrm{Sb}_{5}$ exhibits fullygapped multiband superconductivity.

The low temperature exponential behavior of $\Delta \lambda(T)$ provides evidence for nodeless superconducitivty, while the extracted small gap and analysis of both the superfluid density and the electronic specific heat evidence multiband superconductivity. This is consistent with the presence of multiple Fermi surfaces revealed by angleresolved photoemission spectroscopy measurements and electronic structure calculations [16]. Such a picture is supported by the consideration that the pocket around $\Gamma$ and the Fermi surfaces around $M$ are mainly associated with $\mathrm{Sb} p_{z}$ and $\mathrm{V} d$ orbitals, respectively [24], and are likely associated with superconducting gaps of different sizes. It should be noted that our analyses do not exclude an anisotropic $s$-wave superconducting gap. However, considering that this is a multiband system, and the penetration depth along different directions are similar (Fig. 2(a)), multi-gap superconductivity appears more likely.

Since a circular Fermi surface around $\Gamma$ is present in $\mathrm{CsV}_{3} \mathrm{Sb}_{5}$ [16], all even-parity basis gap functions associated with the $D_{6 h}$ point group would lead to symmetryenforced nodes, except that of the $A_{1 g}$ channel [43]. 
Therefore, the finding of nodeless superconductivity indicates the pairing symmetry of $\mathrm{CsV}_{3} \mathrm{Sb}_{5}$ belongs to the even-parity $A_{1 g}$ representation, or odd-parity nodeless representations. While our results can be described by a superconducting state with two $s$-wave gaps, and is supported by the presence of multiple Fermis surfaces in $\mathrm{CsV}_{3} \mathrm{Sb}_{5}$, our measurements do not rule out some exotic unconventional superconducting states. These include spin-triplet nodeless superconductivity [44], $s^{ \pm}$-pairing as in the iron-based superconductors [45], and pairing with band-mixing as suggested for $\mathrm{CeCu}_{2} \mathrm{Si}_{2}$ [46].

Upon pressure-tuning, superconductivity in $\mathrm{CsV}_{3} \mathrm{Sb}_{5}$ exhibits significant modulations and exhibit at least two superconducting domes [34, 35], suggesting the presence of competing superconducting ground states. As our results evidence a nodeless superconducting state under ambient pressure, whether the competing superconducting state stabilized under pressure remains nodeless or exhibits symmetry-enforced nodes, becomes an important question to be addressed in future works.

In conclusion, the superconducting pairing symmetry of $\mathrm{CsV}_{3} \mathrm{Sb}_{5}$ single crystals is probed through magnetic penetration depth measurements down to $0.07 \mathrm{~K}$. A clear exponential behavior at low temperatures provides evidence for nodeless superconductivity in $\mathrm{CsV}_{3} \mathrm{Sb}_{5}$ under ambient pressure. Temperature dependence of the superfluid density and electronic specific heat can be described by two-gap $s$-wave superconductivity, consistent with the presence of multiple Fermi surfaces in $\mathrm{CsV}_{3} \mathrm{Sb}_{5}$. Our results are inconsistent with pairing with symmetryenforced nodes in $\mathrm{CsV}_{3} \mathrm{Sb}_{5}$, but do not rule out fullygapped unconventional superconductivity.

This work was supported by the National Key R\&D Program of China (No. 2017YFA0303100, No. 2016YFA0300202), the Key R\&D Program of Zhejiang Province, China (2021C01002), the National Natural Science Foundation of China (No. 11974306 and No. 12034017). S.D.W. and B.R.O. gratefully acknowledge support via the UC Santa Barbara NSF Quantum Foundry funded via the Q-AMASE-i program under award DMR-1906325. B.R.O. also acknowledges support from the California NanoSystems Institute through the Elings fellowship program.

* Electronic address: yusong phys@zju.edu.cn

† Electronic address: hqyuan@zju.edu.cn

[1] I. Syozi, Progress of Theoretical Physics 6, 306 (1951), URL https://doi.org/10.1143/ptp/6.3.306.

[2] T.-H. Han, J. S. Helton, S. Chu, D. G. Nocera, J. A. Rodriguez-Rivera, C. Broholm, and Y. S. Lee, Nature 492, 406 (2012), URL https://doi.org/10.1038/nature11659

[3] C. Broholm, R. J. Cava, S. A. Kivelson, D. G. Nocera, M. R. Norman, and
T. Senthil, Science 367, eaay0668 (2020), URL https://doi.org/10.1126/science.aay0668

[4] L. Ye, M. Kang, J. Liu, F. von Cube, C. R. Wicker, T. Suzuki, C. Jozwiak, A. Bostwick, E. Rotenberg, D. C. Bell, et al., Nature 555, 638 (2018), URL https://doi.org/10.1038/nature25987

[5] E. Liu, Y. Sun, N. Kumar, L. Muechler, A. Sun, L. Jiao, S.-Y. Yang, D. Liu, A. Liang, Q. Xu, et al., Nature Physics 14, 1125 (2018), URL https://doi.org/10.1038/s41567-018-0234-5

[6] M. Kang, L. Ye, S. Fang, J.-S. You, A. Levitan, M. Han, J. I. Facio, C. Jozwiak, A. Bostwick, E. Rotenberg, et al., Nature Materials 19, 163 (2019), URL https://doi.org/10.1038/s41563-019-0531-0

[7] Z. Lin, J.-H. Choi, Q. Zhang, W. Qin, S. Yi, P. Wang, L. Li, Y. Wang, H. Zhang, Z. Sun, et al., Physical Review Letters 121 (2018), URL https://doi.org/10.1103/physrevlett.121.096401.

[8] J.-X. Yin, S. S. Zhang, G. Chang, Q. Wang, S. S. Tsirkin, Z. Guguchia, B. Lian, H. Zhou, K. Jiang, I. Belopolski, et al., Nature Physics 15, 443 (2019), URL https://doi.org/10.1038/s41567-019-0426-7

[9] R. Chisnell, J. Helton, D. Freedman, D. Singh, R. Bewley, D. Nocera, and Y. Lee, Physical Review Letters 115 (2015), URL https://doi.org/10.1103/physrevlett.115.147201.

[10] W.-H. Ko, P. A. Lee, and X.-G. Wen, Physical Review B 79 (2009), URL https://doi.org/10.1103/physrevb.79.214502.

[11] S.-L. Yu and J.-X. Li, Physical Review B 85 (2012), URL https://doi.org/10.1103/physrevb.85.144402.

[12] W.-S. Wang, Z.-Z. Li, Y.-Y. Xiang, and Q.H. Wang, Physical Review B 87 (2013), URL https://doi.org/10.1103/physrevb.87.115135.

[13] M. L. Kiesel, C. Platt, and R. Thomale, Physical Review Letters 110 (2013), URL https://doi.org/10.1103/physrevlett.110.126405.

[14] I. I. Mazin, H. O. Jeschke, F. Lechermann, H. Lee, M. Fink, R. Thomale, and R. Valentí, Nature Communications $\mathbf{5}$ (2014), URL https://doi.org/10.1038/ncomms5261.

[15] B. R. Ortiz, L. C. Gomes, J. R. Morey, M. Winiarski, M. Bordelon, J. S. Mangum, I. W. H. Oswald, J. A. Rodriguez-Rivera, J. R. Neilson, S. D. Wilson, et al., Physical Review Materials 3 (2019), URL https://doi.org/10.1103/physrevmaterials.3.094407

[16] B. R. Ortiz, S. M. Teicher, Y. Hu, J. L. Zuo, P. M. Sarte, E. C. Schueller, A. M. Abeykoon, M. J. Krogstad, S. Rosenkranz, R. Osborn, et al., Physical Review Letters 125 (2020), URL https://doi.org/10.1103/physrevlett.125.247002.

[17] B. R. Ortiz, E. Kenney, P. M. Sarte, S. M. L. Teicher, R. Seshadri, M. J. Graf, and S. D. Wilson (2020), arXiv:2012.09097.

[18] Q. Yin, Z. Tu, C. Gong, Y. Fu, S. Yan, and H. Lei (2021), arXiv:2101.10193.

[19] Y.-X. Jiang, J.-X. Yin, M. M. Denner, N. Shumiya, B. R. Ortiz, J. He, X. Liu, S. S. Zhang, G. Chang, I. Belopolski, et al. (2020), arXiv:2012.15709.

[20] H. Zhao, H. Li, B. R. Ortiz, S. M. L. Teicher, T. Park, M. Ye, Z. Wang, L. Balents, S. D. Wilson, and I. Zeljkovic (2021), arXiv:2103.03118.

[21] H. Chen, H. Yang, B. Hu, Z. Zhao, J. Yuan, Y. Xing, G. Qian, Z. Huang, G. Li, Y. Ye, et al. (2021), 
arXiv:2103.09188.

[22] H. X. Li, T. T. Zhang, Y. Y. Pai, C. Marvinney, A. Said, T. Yilmaz, Q. Yin, C. Gong, Z. Tu, E. Vescovo, et al. (2021), arXiv:2103.09769.

[23] H. Tan, Y. Liu, Z. Wang, and B. Yan (2021), arXiv:2103.06325.

[24] X. Feng, K. Jiang, Z. Wang, and J. Hu (2021), arXiv:2103.07097.

[25] S.-Y. Yang, Y. Wang, B. R. Ortiz, D. Liu, J. Gayles, E. Derunova, R. Gonzalez-Hernandez, L. Šmejkal, Y. Chen, S. S. P. Parkin, et al., Science Advances 6, eabb6003 (2020), URL https://doi.org/10.1126/sciadv.abb6003.

[26] E. M. Kenney, B. R. Ortiz, C. Wang, S. D. Wilson, and M. J. Graf (2020), arXiv:2012.04737.

[27] F. H. Yu, T. Wu, Z. Y. Wang, B. Lei, W. Z. Zhuo, J. J. Ying, and X. H. Chen (2021), arXiv:2102.10987.

[28] Y. Wang, S. Yang, P. K. Sivakumar, B. R. Ortiz, S. M. L. Teicher, H. Wu, A. K. Srivastava, C. Garg, D. Liu, S. S. P. Parkin, et al. (2020), arXiv:2012.05898.

[29] Z. Liang, X. Hou, W. Ma, F. Zhang, P. Wu, Z. Zhang, F. Yu, J. J. Ying, K. Jiang, L. Shan, et al. (2021), arXiv:2103.04760.

[30] D. J. Scalapino, Reviews of Modern Physics 84, $1383 \quad$ (2012), URL https://doi.org/10.1103/revmodphys.84.1383.

[31] G. R. Stewart, Advances in Physics 66, 75 (2017), URL https://doi.org/10.1080/00018732.2017.1331615.

[32] D. J. V. Harlingen, Reviews of Modern Physics 67, $515 \quad$ (1995), URL https://doi.org/10.1103/revmodphys.67.515.

[33] C. C. Tsuei and J. R. Kirtley, Reviews of Modern Physics 72, 969 (2000), URL https://doi.org/10.1103/revmodphys.72.969.

[34] C. C. Zhao, L. S. Wang, W. Xia, Q. W. Yin, J. M. Ni, Y. Y. Huang, C. P. Tu, Z. C. Tao, Z. J. Tu, C. S. Gong, et al. (2021), arXiv:2102.08356.

[35] K. Y. Chen, N. N. Wang, Q. W. Yin, Z. J. Tu, C. S. Gong, J. P. Sun, H. C. Lei, Y. Uwatoko, and J. G. Cheng
(2021), arXiv:2102.09328.

[36] F. Du, S. Luo, B. R. Ortiz, Y. Chen, W. Duan, D. Zhang, X. Lu, S. D. Wilson, Y. Song, and H. Yuan (2021), 2102.10959.

[37] C. T. Van Degrift, Rev. Sci. Instrum. 46, 599 (1975), URL https://doi.org/10.1063/1.1134272.

[38] E. M. E. Chia, Ph.D. thesis, University of Illinois at UrbanaChampaign (2004), URL http://research.physics.illinois.edu/Publications/theses/co

[39] R. Prozorov, R. W. Giannetta, A. Carrington, and F. M. Araujo-Moreira, Phys. Rev. B 62, 115 (2000), URL https://link.aps.org/doi/10.1103/PhysRevB.62.115

[40] T. P. Orlando, E. J. McNiff, S. Foner, and M. R. Beasley, Physical Review B 19, 4545 (1979), URL https://doi.org/10.1103/physrevb.19.4545

[41] H. Q. Yuan, D. F. Agterberg, N. Hayashi, P. Badica, D. Vandervelde, K. Togano, M. Sigrist, and M. B. Salamon, Physical Review Letters 97 (2006), URL https://doi.org/10.1103/physrevlett.97.017006.

[42] F. Gross, B. S. Chandrasekhar, D. Einzel, K. Andres, P. J. Hirschfeld, H. R. Ott, J. Beuers, Z. Fisk, and J. L. Smoth, Z. Phys. B: Condens. Matter 64, 175 (1986), URL https://doi.org/10.1007/BF01303700.

[43] M. Sigrist and K. Ueda, Reviews of Modern Physics 63, 239 (1991), URL https://doi.org/10.1103/revmodphys.63.239.

[44] R. Balian and N. R. Werthamer, Physical Review 131, $1553 \quad$ (1963), URL https://doi.org/10.1103/physrev.131.1553

[45] P. J. Hirschfeld, M. M. Korshunov, and I. I. Mazin, Reports on Progress in Physics 74, 124508 (2011), URL https://doi.org/10.1088/0034-4885/74/12/124508.

[46] G. Pang, M. Smidman, J. Zhang, L. Jiao, Z. Weng, E. M. Nica, Y. Chen, W. Jiang, Y. Zhang, W. Xie, et al., Proceedings of the National Academy of Sciences 115, 5343 (2018), URL https://doi.org/10.1073/pnas.1720291115 